\title{
BIBLIOGRAPHY
}

1. E. Miot, Question 3825, L'intermédiare des Mathématiciens vol. 18 (1911) p. 49.

2. A. Cunningham, Solution de question 3825, L'intermédiaire des Mathématiciens vol. 18 (1911) pp. 210-213.

3. L. E. Dickson, History of the theory of numbers, vol. 2, Diophantine analysis, Washington, 1920.

University of Pennsyluania

\section{SQUARE ROOTS IN LOCALLY EUCLIDEAN GROUPS}

\section{A. M. GLEASON}

A possible attack on the fifth problem of Hilbert is to demonstrate the existence of one-parameter subgroups in any locally Euclidean group. ${ }^{1}$ It is known that, provided there are no "small" subgroups, some one-parameter subgroups exist. One would like to prove, however, that in a suitable neighborhood of the identity, every element is on one and only one one-parameter subgroup. If this is true, it is possible to extract square roots (that is, solve $x^{2}=a$ for given $a$ ) uniquely in this neighborhood, and the sequence of successive square roots $a, a^{1 / 2},\left(a^{1 / 2}\right)^{1 / 2}, \cdots$ converges to the identity. Conversely, it is easily seen that, if unique square roots exist, and if the sequence of square roots converge to the identity, then the one-parameter subgroups can be found. In this paper we give a new proof ${ }^{2}$ that square roots exist in a suitable neighborhood of the identity and show, in addition, that either they are unique or small subgroups exist.

Throughout this paper we shall deal only with locally Euclidean topological groups of dimension $n$; consequently we may speak of an $n$-cell neighborhood of a point $p$, meaning a homeomorphic image of a Euclidean $n$-simplex containing the point $p$ in its interior.

The proofs of Theorems 1 and 2 use the group property so sparingly that they can easily be restated as theorems on the involutions of a manifold.

THEOREM 1. In a locally Euclidean group there exists a neighborhood

Received by the editors March 26, 1948.

1 The arguments outlined here are those of B. von Kerekjarto in his paper Geometrische Theorie der zweigliedrigen kontinuierlichen Gruppen, Abh. Math. Sem. Hamburgischen Univ. vol. 8 (1930) pp. 107-114.

2 Cf. B. de Kerekjarto, Sur l'existence de racines carrées dans les groupes continus, C. R. Acad. Sci. Paris vol. 193 (1931) pp. 1384-1385. 


\section{of the identity containing no elements of order two. ${ }^{3}$}

Proof. Let $C_{n}$ be an $n$-cell neighborhood of the identity, e. We can easily construct a sequence, $C_{n-1}, \cdots, C_{0}$ (the subscripts are merely indices and do not refer to dimension), of $n$-cell neighborhoods of $e$ such that $C_{k}^{2} \subset C_{k+1}(k=0,1, \cdots, n-1)$. We claim that there are no elements of order two in $C_{0}$.

Suppose, on the contrary, that there is an element $a \in C_{0}$ such that $a \neq e$ and $a^{2}=e$. We shall construct by induction a singular $n$-sphere in the cell $C_{n}$ (that is, a continuous function $f$ mapping the unit sphere $S^{n}$ of $n+1$ dimensional space into the cell $C_{n}$ ) satisfying the relation

$$
T \circ f=f \circ A,
$$

where $T$ is the group translation $T(x)=a x$, and $A$ is the antipodal map of $S^{n}$ into itself. $S^{n}$ contains in a natural fashion the sequence of spheres $S^{0}, S^{1}, \cdots, S^{n-1}, S^{n}$, each being the "equator" of the next. Define $f$ on $S^{0}$ by letting $e$ and $a$ be the images of the two points of $S^{0}$. Evidently (1) holds and $f\left(S^{0}\right) \subset C_{0}$. Suppose that $k<n$, and that $f$ is defined on $S^{k}$ and $f\left(S^{k}\right) \subset C_{k}$. Since $C_{k}$ is aspherical in all dimensions we can extend $f$ over one hemisphere of $S^{k+1}$, the image being in $C_{k}$. Then we may use the relation (1) to define $f$ over the other hemisphere and, since $T\left(C_{k}\right) \subset C_{k+1}$, we shall have $f\left(S^{k+1}\right) \subset C_{k+1}$. Thus we obtain the desired singular $n$-sphere. However, the Ulam-Borsuk theorem asserts, for any singular $n$-sphere in an $n$-cell, that there is a point $x$ for which $f(x)=f \circ A(x)$. By (1) we have $T \circ f(x)=f(x)$, a contradiction since $T$ has no fixed points.

Theorem 2. If $C$ is a Euclidean n-cell containing the identity, $e$, then there exists a singular $(n-1)$-sphere $f$ in $C-\{e\}$ such that

$$
I \circ f=f \circ A,
$$

where $I$ is the inversive mapping, $I(x)=x^{-1}$. Moreover, if $f$ is any such singular (n-1)-sphere, and if $C \cup C^{-1} \subset D$, where $D$ is an n-cell containing no elements of order two, then $f$ is an essential singular sphere in $C-\{e\}$.

Proof. We proceed as in Theorem 1, choosing first the $n$-cells $C_{n-1}=C, C_{n-2}, \cdots, C_{0}$ where each is a neighborhood of the identity and $C_{k} \cup C_{k}{ }^{-1} \subset C_{k+1} C_{k}{ }^{-1} \subset C_{k+1}$. We can define $f$ on $S^{0}$ so that (2) holds and $f\left(S^{0}\right) \subset C_{0}-\{e\}$. Since the sets $C_{k}-\{e\}$ are aspherical in the dimensions $0,1, \cdots, n-2$ the inductive extension of $f$ can be car-

${ }^{3}$ This theorem was first given by M. H. A. Newman, $A$ theorem on periodic transformations of spaces, Quart. J. Math. Oxford Ser. vol. 2 (1931) pp. 1-8. 
ried through $n-1$ steps, using (2) in place of (1), to give the required singular $(n-1)$-sphere.

Now suppose that the cell $D$ exists and that $f$ is a singular $(n-1)$ sphere in $C-\{e\}$ satisfying (2). If $f$ is not essential, extend $f$ over a hemisphere of $S^{n}$ and complete the extension over $S^{n}$ by the relation (2). Then $e \notin f\left(S^{n}\right) \subset D$ and, the latter being an $n$-cell, the UlamBorsuk theorem tells us that, for some $x, f(x)=f \circ A(x)$. By (2) we have $f(x)=[f(x)]^{-1}$, but $D$ contains no elements of order two and $f(x) \neq e$; we have found a contradiction.

COROLlaRy. The degree of the inversive mapping about the identity is $(-1)^{n}$.

Proof. With $f$ as in the theorem above we have $\partial I \times \partial f=\partial(I \circ f)$ $=\partial(f \circ A)=\partial f \times \partial A$, and we know that $\partial f \neq 0$. Hence $\partial I=\partial A=(-1)^{n}$.

ThEOREM 3. If $N$ is a given neighborhood of the identity, there is a neighborhood $M$ of the identity such that every element of $M$ has a square root in $N$.

Proof. We can choose $n$-cell neighborhoods of the identity, $B, C$, and $D$, such that $D \subset N, D$ contains no elements of order two, $C \cup C^{-1}$ $\subset D$, and $B^{2} \subset C$. By Theorem 2 we can construct a singular $(n-1)$ sphere $f$ in $B-\{e\}$ satisfying (2). Let $Q$ be the quadratic mapping $Q(x)=x^{2}$. Since $I$ and $Q$ permute, $I \circ Q \circ f=Q \circ I \circ f=Q \circ f \circ A$. Hence $Q \circ f$ is a singular $(n-1)$-sphere in $C-\{e\}$ satisfying (2). Applying the second part of Theorem 2, $Q \circ f$ is essential in $C-\{e\}$ and this implies that $Q$ is an essential mapping of $B-\{e\}$ into $C-\{e\}$. Hence every point of a sufficiently small neighborhood $M$ of the identity is the image under $Q$ of some point of $B$; that is, every point of $M$ has a square root in $B \subset N$.

COROLLARY. The same theorem is true for roots of any order.

Definition. A topological group is said to have small subgroups if every neighborhood of the identity contains a nontrivial subgroup (that is, a subgroup with more than one element).

THEOREM 4. If a locally Euclidean group does not have small subgroups, then there are neighborhoods $M$ and $N$ of the identity such that every element of $M$ has a unique square root in $N$.

Proof. Let $C$ and $N$ be $n$-cell neighborhoods of the identity such that $C$ contains no nontrivial subgroups, and $N N^{-1} N \subset C$. Since $C$-Int $N$ is compact and contains no elements of order two, its image under $Q$ is closed and does not contain $e$. With the aid of 
Theorem 3 we can choose a neighborhood $M$ of the identity such that $M \cap Q(C-$ Int $N)$ is void and such that every element of $M$ has at least one square root in $N$. The construction shows that, if $a \in M$, any square root of a lying in $C$ is actually in $N$.

Suppose that $x \in N$ and $y \in N$ are distinct square roots of $a \in M$. It is clear (without using the hypothesis about small subgroups) that $x$ and $y$ do not commute, for then $z=x y^{-1}$ would be an element of order two. We shall show that all powers of $z$ are in $C$. Since $x^{2}=y^{2}$, $y^{-1} x \cdot x y^{-1}=y^{-1} y^{2} y^{-1}=e$, hence $z^{-1}=y^{-1} x$, and $z x=x z^{-1}$. Then $z^{m} x \cdot z^{m} x$ $=x z^{-m} z^{m} x=a$; that is, all the elements $z^{m} x$ are square roots of $a$. We shall show by induction that all of these elements are in $N$. If $m>0$ and $z^{m-1} x \in N, z^{m} x=\left(z^{m-1} x\right) y^{-1} x \in N N^{-1} N \subset C$, hence $z^{m} x \in N$. Then $z^{ \pm m} \in N N^{-1} \subset C$ for all $m$, and we have found a nontrivial subgroup entirely contained in $C$, a contradiction.

HARVARD UNIVERSITY 